\title{
Loss of Doc2-Dependent Spontaneous Neurotransmission Augments Glutamatergic Synaptic Strength
}

\author{
DDenise M.0. Ramirez, ${ }^{1 *}$ Devon C. Crawford, ${ }^{2 \star}$ Natali L. Chanaday, ${ }^{2}$ Brent Trauterman, ${ }^{2}$ Lisa M. Monteggia, ${ }^{2}$ \\ and ${ }^{-E g e}$ T. Kavalali ${ }^{2,3}$ \\ ${ }^{1}$ Department of Neurology and Neurotherapeutics, ${ }^{2}$ Department of Neuroscience, and ${ }^{3}$ Department of Physiology, University of Texas Southwestern \\ Medical Center, Dallas, Texas 75390
}

Action potential-evoked vesicle fusion comprises the majority of neurotransmission within chemical synapses, but action potentialindependent spontaneous neurotransmission also contributes to the collection of signals sent to the postsynaptic cell. Previous work has implicated spontaneous neurotransmission in homeostatic synaptic scaling, but few studies have selectively manipulated spontaneous neurotransmission without substantial changes in evoked neurotransmission to study this function in detail. Here we used a quadruple knockdown strategy to reduce levels of proteins within the soluble calcium-binding double C2 domain (Doc2)-like protein family to selectively reduce spontaneous neurotransmission in cultured mouse and rat neurons. Activity-evoked responses appear normal while both excitatory and inhibitory spontaneous events exhibit reduced frequency. Excitatory miniature postsynaptic currents (mEPSCs), but not miniature inhibitory postsynaptic currents (mIPSCs), increase in amplitude after quadruple knockdown. This increase in synaptic efficacy correlates with reduced phosphorylation levels of eukaryotic elongation factor 2 and also requires the presence of elongation factor 2 kinase. Together, these data suggest that spontaneous neurotransmission independently contributes to the regulation of synaptic efficacy, and action potential-evoked and spontaneous neurotransmission can be segregated at least partially on a molecular level.

Key words: Doc2; spontaneous neurotransmitter release; synaptic scaling

Significance Statement

Action potential-evoked and spontaneous neurotransmission have been observed in nervous system circuits as long as methods have existed to measure them. Despite being well studied, controversy still remains about whether these forms of neurotransmission are regulated independently on a molecular level or whether they are simply a continuum of neurotransmission modes. In this study, members of the Doc2 family of presynaptic proteins were eliminated, which caused a reduction in spontaneous neurotransmission, whereas action potential-evoked neurotransmission remained relatively normal. This protein loss also caused an increase in synaptic strength, suggesting that spontaneous neurotransmission is able to communicate independently with the postsynaptic neuron and trigger downstream signaling cascades that regulate the synaptic state.

\section{Introduction}

Action potential-evoked neurotransmitter release is vital to the flow of information through neuronal circuits, but less is known

Received Feb. 14, 2017; revised May 2, 2017; accepted May 13, 2017.

Author contributions: D.M.O.R., D.C.C., N.L.C., L.M.M., and E.T.K. designed research; D.M.O.R., D.C.C., N.L.C., and B.T. performed research; B.T. contributed unpublished reagents/analytic tools; D.M.O.R., D.C.C., and E.T.K. analyzed data; D.M.O.R., D.C.C., and E.T.K. wrote the paper.

${ }^{*} D$.M.O.R. and D.C.C. contributed equally to this work.

This work was supported by National Institutes of Health Grants F32MH102915 to D.C.C., R01MH070727 to L.M.M., R01MH066198 to E.T.K., Brain and Behavior Research Foundation to D.M.O.R., L.M.M., and E.T.K., and the International Mental Health Research Organization to L.M.M. The work described in this article was performed while all authors were employed at the University of Texas Southwestern Medical Center. The opinions expressed in this article are the authors' own and do not reflect the view of the National Institutes of Health, the Department of Health and Human Services, or the United States Government. The authors declare no competing financial interests. We thank members of the E.T.K. and L.M.M. laboratories for insightful discussions.

The authors declare no competing financial interests. about the signals communicated by spontaneous neurotransmitter release, which occurs independently of the presence of action potentials. Spontaneous neurotransmission has well-studied roles in synapse development (McKinney et al., 1999; Andreae et al., 2012) and homeostatic synaptic plasticity (Sutton et al., 2006; Aoto et al., 2008), but most of these studies have manipulated action potential-evoked neurotransmission alongside spontaneous neurotransmission rather than separately. The simultaneous block of both forms of neurotransmission makes it difficult to clarify whether the effects are induced by additive effects of these forms of vesicle release.

Correspondence should be addressed to Dr. Ege T. Kavalali, Department of Neuroscience, University of Texas Southwestern Medical Center, 5323 Harry Hines Blvd, Dallas, TX75390-9111. E-mail: Ege.Kavalali@UTSouthwestern.edu. DOI:10.1523/JNEUROSCI.0418-17.2017

Copyright $\odot 2017$ the authors $\quad 0270-6474 / 17 / 376224-07 \$ 15.00 / 0$ 
Although prior work has attempted to tease apart the effects of these forms of neurotransmission using pharmacological and electrical manipulations (Sutton et al., 2006; Fong et al., 2015), molecular components of the presynaptic structure have also been implicated in selective control of different forms of neurotransmission (Crawford and Kavalali, 2015; Kavalali, 2015). Exploitation of this system could help clarify the differential functions and consequences of evoked and spontaneous neurotransmission. In a recent study (Crawford et al., 2017), we knocked down the vesicular soluble NSF attachment protein receptor (v-SNARE) proteins Vps10p-tail-interactor-1a (vtila) and vesicle-associated membrane protein 7 (VAMP7) to selectively reduce spontaneous neurotransmission without producing impairment of evoked neurotransmission. Vtila and VAMP7 are located in synaptic vesicles and have both been implicated in selectively driving spontaneous but not synchronously evoked neurotransmission (Antonin et al., 2000; Muzerelle et al., 2003; Hua et al., 2011; Ramirez et al., 2012; Bal et al., 2013). We found that loss of these SNARE proteins reduces spontaneous neurotransmission, but not synchronous action potential-evoked neurotransmission, while inducing upward synaptic scaling of glutamatergic responses, which suggests that this form of neurotransmission signals independently to the postsynaptic compartment. This study did not, however, clarify whether these effects were due to the general manipulation of spontaneous neurotransmission or to the specific modulation of vtila- and VAMP7-dependent neurotransmission. To clarify this question, other molecules that selectively alter spontaneous neurotransmission need to be tested.

One class of proteins thought to participate in spontaneous but not fast evoked neurotransmitter release is the double C2 domain (Doc2) family. The Doc2-like protein family comprises four isoforms (Doc2A, Doc2B, Doc2G, and rabphilin), some of which have been recently identified as specific regulators of spontaneous (Groffen et al., 2010; Pang et al., 2011) or asynchronous (Sakaguchi et al., 1999; Yao et al., 2011) neurotransmitter release. These proteins may act as soluble $\mathrm{Ca}^{2+}$ sensors in this process (Groffen et al., 2010; Yao et al., 2011; Gaffaney et al., 2014; but see Pang et al., 2011). In a prior study in which four Doc2-like proteins were knocked down, the amplitude of excitatory spontaneous events was nonsignificantly increased alongside a decrease in their frequency (Pang et al., 2011), perhaps suggesting that synaptic strength is altered in a compensatory fashion. Additional work is required to clarify this result and the underlying mechanisms involved.

To test whether altered spontaneous neurotransmission caused by loss of Doc2-like proteins induces changes in synaptic strength, we also used a quadruple knockdown strategy in cultured hippocampal neurons. This manipulation successfully reduced the frequency of spontaneous events without appreciably altering fast evoked neurotransmission. We detected increased strength of excitatory neurotransmission, but not inhibitory neurotransmission, in neurons with reduced levels of Doc2like proteins, and this effect was rescued by Doc2B overexpression. Loss of Doc2-like proteins also reduced phosphorylation levels of eukaryotic elongation factor 2 (eEF2), and scaling was not induced in eEF2 kinase knock-out neurons, suggesting that Doc2-dependent neurotransmission requires eEF2 signaling to control synaptic strength.

\section{Materials and Methods}

Cell culture. Cultures of dissociated hippocampal neurons were prepared as previously described (Kavalali et al., 1999). Briefly, hippocampi were dissected from male and female postnatal day 0-3 (P0-P3) Sprague Daw-
A
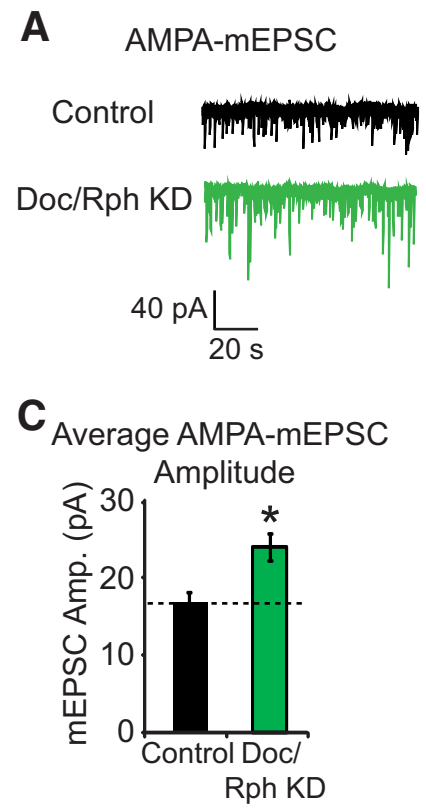

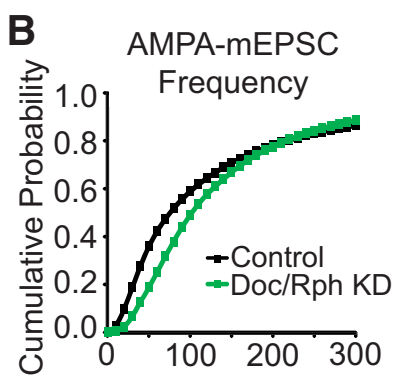

D

Inter-event Interval (ms)

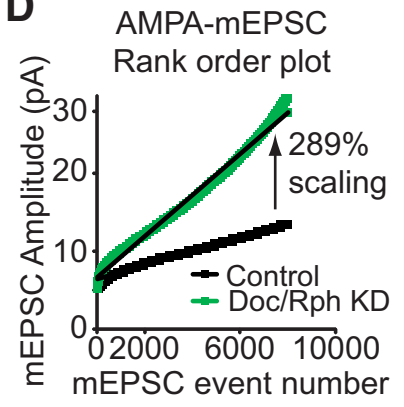

Figure 1. Loss of Doc2-like proteins decreases the frequency but increases the amplitude of spontaneous excitatory events. $A$, Representative traces of AMPA-mEPSC recordings in GFPinfected (Control) or Doc2A, Doc2B, Doc2G, and Rabphilin quadruple knockdown (Doc/Rph KD) neurons in rat hippocampal cultures. $\boldsymbol{B}$, Cumulative probability histograms of AMPA-mEPSC interevent intervals from Control and Doc/Rph KD neurons. AMPA-mEPSC interevent interval is significantly increased, suggesting a decreased frequency, in Doc/Rph knockdown neurons compared with control neurons ( $p=0.0001$; Kolmogorov-Smirnov test; data collected from 9-30 neurons per condition from two to six independent cultures). C, Average AMPA-mEPSC amplitudes from Control and Doc/Rph KD neurons. Doc/Rph KD neurons exhibit significantly increased AMPA-mEPSC amplitudes ( $p=0.036$; Student's $t$ test; $n=9-30$ neurons from two or six independent cultures). $\boldsymbol{D}$, Rank-order plot of AMPA-mEPSC amplitudes from Control and Doc/Rph KD neurons analyzed in $C$. The slopes of the linear fits of the two curves indicate a multiplicative increase ( $289 \%$ scaling) of AMPA-mEPSC amplitudes in Doc/Rph KD neurons compared with Control. ${ }^{*}, p<0.05$.

ley rat pups (strain code 001; Charles River Laboratories) or eukaryotic elongation factor 2 kinase (eEF2K) knock-out or sibling wild-type mouse pups (gift of Dr. Alexey Ryazanov). After trypsinization (10 mg/ml tryp$\sin )$ at $37^{\circ} \mathrm{C}$ for $10 \mathrm{~min}$, neurons were mechanically dissociated and plated onto glass coverslips coated in Matrigel (BD Biosciences) for rat cultures and poly-D-lysine for mouse cultures. At DIV 1, $4 \mu \mathrm{M}$ cytosine arabinoside was added, which was reduced to $2 \mu \mathrm{M}$ at DIV 4 . All experiments were performed when the cultures were DIV 14-21.

Lentiviral preparation. We used a short hairpin RNA knockdown strategy to reduce levels of four Doc2 family proteins (Doc2A, Doc2B, Doc2G, and rabphilin; designated Doc/Rph KD) as described previously (Pang et al., 2011). Two constructs were used for this purpose: KD43 and KD136. We also used a Doc2B rescue construct (KD136 + Doc2B rescue), and all of the Doc2 constructs were kind gifts of Drs. Z. Pang and T. Sudhof at Stanford University. Transfection of human embryonic kidney (HEK) 293-T cells with the Doc2 plasmids and the viral packaging and coating plasmids (pRSV-Rev, pPRE-MALG, and pVSVG) was accomplished using Fugene 6 (Roche Applied Science). After 3 d, the HEK 293-T-conditioned medium was harvested and added to the neuronal culture medium at DIV 4, and infection frequencies consistently approached $100 \%$. Control neurons were from the same cultures but either not infected or infected with the empty L307 vector expressing soluble GFP.

Electrophysiology. Whole-cell patch-clamp recordings were performed on hippocampal pyramidal neurons bathed in a modified Tyrode's solution at pH 7.4 containing the following (in $\mathrm{mM}$ ): $150 \mathrm{NaCl}, 4 \mathrm{KCl}, 2 \mathrm{MgCl}_{2}, 2$ $\mathrm{CaCl}_{2}, 10$ glucose, and $10 \mathrm{HEPES}$. Internal pipette solution at $300 \mathrm{mOsm}$ and $7.3 \mathrm{pH}$ contained the following (in mM): $115 \mathrm{Cs}-\mathrm{MeSO}_{3}, 10 \mathrm{CsCl}, 5 \mathrm{NaCl}, 10$ HEPES, 0.6 EGTA, 20 tetraethylammonium-Cl, 4 Mg-ATP, $0.3 \mathrm{Na}_{3} \mathrm{GTP}$, 
A

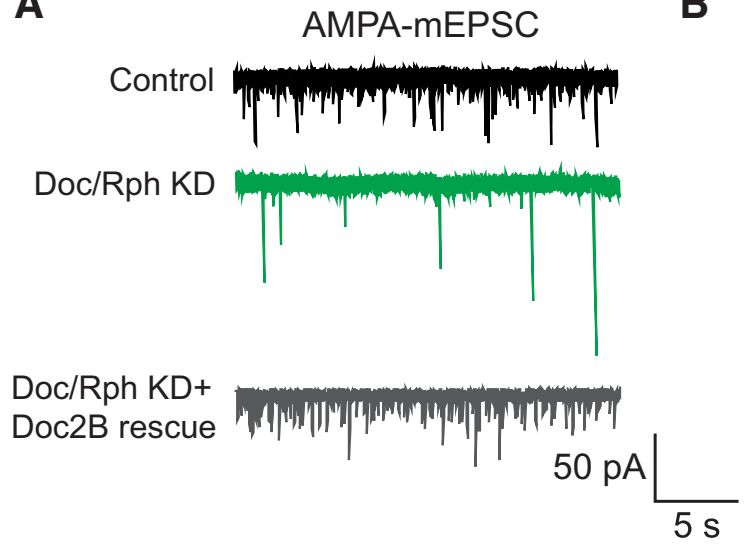

B
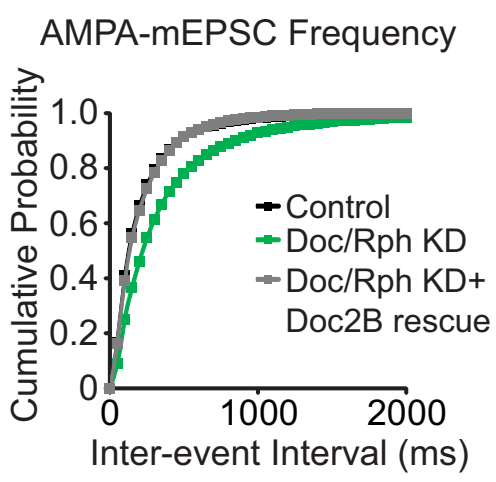

C
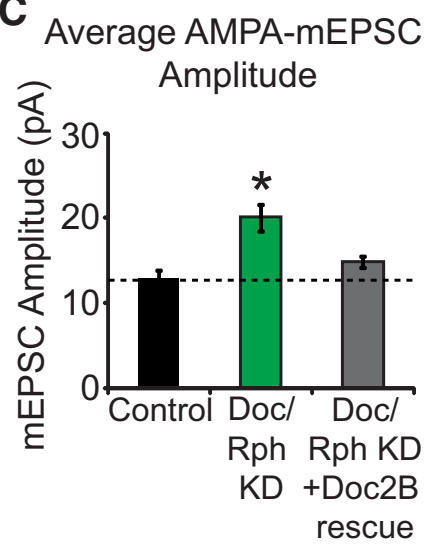

Figure 2. shRNA-resistant Doc2B rescues AMPA-mEPSC parameters altered by loss of Doc2-like proteins. $A$, Representative traces of AMPA-mEPSC recordings in GFP-infected (Control), Doc/Rph $\mathrm{KD}$, or Doc/Rph KD with coexpression of shRNA-resistant Doc2B (Doc2B rescue) neurons in rat hippocampal cultures. B, Cumulative probability histograms of AMPA-mEPSC interevent intervals from Control, Doc/Rph KD, and Doc/Rph KD + Doc2B rescue neurons. AMPA-mEPSC interevent interval is significantly increased in Doc/Rph knockdown neurons but not in Doc/Rph KD + Doc $2 B$ rescue neurons compared with Control (Control vs Doc/Rph KD: Bonferroni-corrected, $p<0.05$; Control vs Doc/Rph KD + Doc2B rescue: uncorrected, $p>0.05$; Kolmogorov-Smirnov test). Data were collected from 8-19 neurons per condition from two to five independent cultures. C, Average AMPA-mEPSC amplitudes from Control, Doc/Rph KD, and Doc/Rph KD + Doc2B rescue neurons analyzed in $\boldsymbol{B}$. A significant increase in AMPA-mEPSC amplitude was observed in Doc/Rph KD neurons but not in Doc/Rph KD + Doc2B neurons compared with Control (Control vs Doc/Rph KD: Bonferroni-corrected, $p<0.001$; Control vs Doc/Rph KD + Doc2B rescue: uncorrected, $p=0.21$; ANOVA followed by Student's $t$ test). ${ }^{*}$, Bonferroni corrected $p<0.05$.

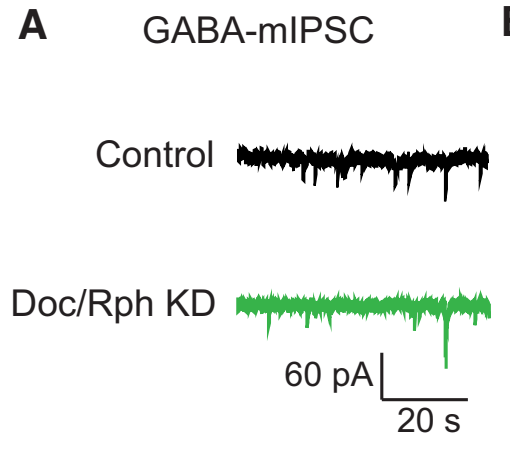

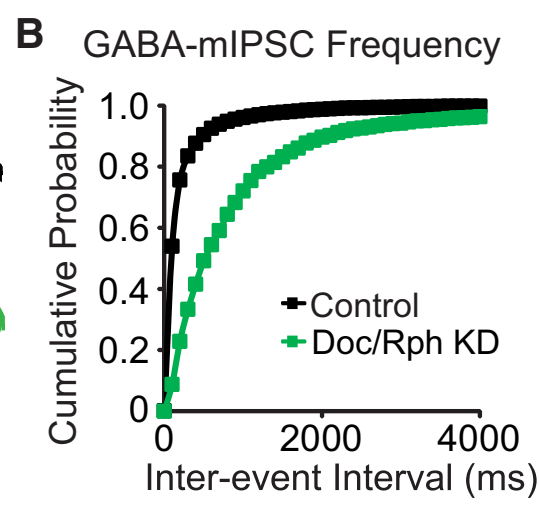

\section{Average GABA-mIPSC Amplitude}

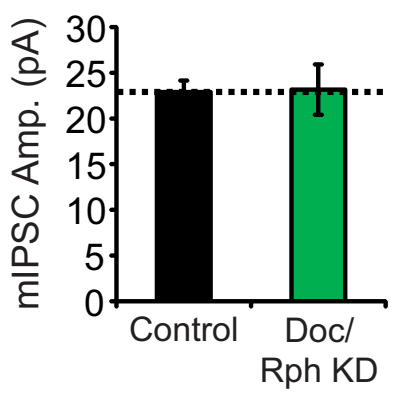

Figure 3. Loss of Doc2-like proteins decreases the frequency of spontaneous inhibitory events but does not alter their amplitudes. $\boldsymbol{A}$, Representative traces of GABA-mIPSC recordings in Control or Doc/Rph KD neurons in rat hippocampal cultures. B, Cumulative probability histograms of GABA-mIPSC interevent intervals from Control and Doc/Rph KD neurons. GABA-mIPSC interevent interval is significantly increased, suggesting a decreased frequency, in Doc/Rph knockdown neurons compared with control neurons ( $p=0.0001$; Kolmogorov-Smirnov test; data collected from 8 neurons per condition from three independent cultures). $\boldsymbol{C}$, Average GABA-mIPSC amplitudes from Control and Doc/Rph KD neurons analyzed in $\boldsymbol{B}$. Doc/Rph KD neurons do not exhibit significantly different GABA-mIPSC amplitudes ( $p=0.94$; Student's $t$ test).

$\mathrm{pH}$ 7.35, and 10 QX-314 [N-(2,6-dimethylphenylcarbamoylmethyl)triethylammonium bromide]. During experiments measuring $\alpha$-amino-3hydroxy-5-methyl-4-isoxazolepropionic acid receptor-dependent miniature EPSCs (AMPA-mEPSCs), $1 \mathrm{~mm}$ TTX, $50 \mathrm{~mm}$ picrotoxin, and $50 \mathrm{~mm}$ DL-AP5 or D-AP5 were added to the bath solution. To measure GABA receptor-dependent miniature inhibitory PSCs (GABA-mIPSCs), $1 \mathrm{~mm}$ TTX, $50 \mathrm{~mm}$ AP5, and $10 \mathrm{~mm}$ CNQX disodium salt hydrate were added to the bath solution. During experiments measuring spontaneous network activity in the cultures, $50 \mathrm{~mm}$ picrotoxin was added to the bath solution. Stimulation-evoked EPSCs were induced $30 \mathrm{~s}$ apart with parallel bipolar electrodes (FHC) immersed in the bath solution, which contained $50 \mathrm{~mm}$ picrotoxin and $50 \mathrm{~mm}$ D-AP5. For paired-pulse experiments, paired stimulation with a 50 or $100 \mathrm{~ms}$ interval was applied every $30 \mathrm{~s}$. Data were acquired using a MultiClamp 700B or AxoPatch 200B amplifier and Clampex 9.0 software (Molecular Devices), filtered at $2 \mathrm{kHz}$, and sampled at $200 \mu$ s.

Western blotting. Western blotting for total and phospho-eEF2 levels was performed as previously described (Crawford et al., 2017). Briefly, neuronal cultures were harvested in $2 \times$ Laemmli Sample Buffer (\#1610737; Bio-Rad) with 5\% 2-mercaptoethanol, sonicated for $30 \mathrm{~s}$, and boiled for $5 \mathrm{~min}$ at $95^{\circ} \mathrm{C}$. Immunoblotting was then performed according to the manufacturer's protocol (Odyssey Infrared Imaging System, LiCor Biosciences) using the following primary antibodies: anti-total eEF2 (1:750 dilution) and anti-phospho-eEF2 (1:1000 dilution) rabbit polyclonal antibodies (Cell Signaling Technology) and anti-Rab-GDI mouse monoclonal antibody (1:10,000 dilution; Synaptic Systems). Secondary antibodies were IRDye-680-conjugated goat anti-rabbit (\#926-32221; Li-Cor Biosciences) and IRDye-800-conjugated goat anti-mouse secondary antibodies (\#926-32210; Li-Cor Biosciences) used at 1:10,000 dilution. A Li-Cor Odyssey machine was then used to scan the membranes at $169 \mathrm{~mm}$ resolution and high quality with intensity values kept in the 3-6 range. The application software was then used to analyze band intensities within the TIF image before export to Excel (Microsoft).

$q R T-P C R$. RNA was extracted from DIV 14-21 cultured neurons using the PureLink RNA Mini Kit (\#12183018A; Ambion from Thermo Fisher Scientific). The qRT-PCR was performed according to the manufacturer's protocol (TaqMan One-Step RT-PCR with Taqman Universal Master Mix II, no UNG; Applied Biosystems from Thermo Fisher Scientific). Transcript levels of Doc2A (Rn00576041_g1), Doc2B (Rn00579752_m1), Doc2G (Rn01474496_m1), and Rph3a (Rn00591716_m1) were assessed using manufacturer primer sets and normalized to levels of the endogenous control transcript GAPDH (Mm99999915_g1; Thermo Fisher Scientific). 
Luminal synaptotagmin antibody uptake with phospho-eEF2 immunostaining. Antibody against the luminal domain of synatotagmin1 was loaded into neurons as previously described (Crawford et al., 2017). Briefly, neurons were treated for $5 \mathrm{~min}$ at room temperature in electrophysiological bath solution containing $1 \mathrm{~mm}$ TTX followed by $15 \mathrm{~min}$ in the same solution, except with 1:100 dilution of mouse monoclonal antibody against the luminal epitope of synaptotagmin 1 added (Synaptic Systems). After fixation in PBS containing 4\% PFA, neurons were processed for phospho-eEF2 immunocytochemistry as previously described (Ramirez et al., 2008). Primary antibody against phospho-eEF2 (rabbit polyclonal antibody; Cell Signaling Technology) was used at 1:500 dilution. Secondary antibodies for detecting synaptotagmin 1 and phosphoeEF2 were Alexa-488-conjugated goat anti-mouse antibody (\#A-11029; Thermo Fisher Scientific) and Alexa-594-conjugated goat anti-rabbit antibody (\#A-11037; Thermo Fisher Scientific), respectively, used at 1:1000 dilution. Imaging was performed on an LSM510 confocal using LSM 5 software (Carl Zeiss), and 15-30 puncta of constant diameter containing both synaptotagmin 1- and phospho-eEF2-positive regions (presumed to indicate presynaptic and postsynaptic regions, respectively) were selected per image. The absolute fluorescence intensities of the red and green puncta were measured and calculated offline using LSM 5 software.

Reagents. All reagents were from Sigma-Aldrich unless otherwise specified.

Statistics. Statistical analyses were performed using Excel 2010 (Microsoft) and SigmaPlot 12 (Systat Software) software. For two independent conditions of approximately normally distributed data with similar SEs, an unpaired two-tailed $t$ test was used unless otherwise stated, whereas for non-normally distributed data a Mann-Whitney $U$ test was used unless otherwise stated. Statistical significance was defined as $p<$ 0.05 , and one-way ANOVA followed by Bonferroni correction for multiple comparisons was applied to determine significance in datasets with more than two groups. Sample sizes were not statistically predetermined but conform to similar studies. All results are presented as mean \pm SEM unless otherwise stated.

\section{Results}

\section{Knockdown of Doc2 isoforms triggers excitatory} synaptic scaling

To date, the number of specific molecules identified that participate preferentially in different modes of neurotransmission is quite limited, with vtila and VAMP7 representing the only SNAREs known to drive vesicle release at rest without substantial effects on fast synchronous release (Hogins et al., 2011; Raingo et al., 2012; Adachi and Monteggia, 2014; Crawford et al., 2017). Recent work has also identified members of the double C2 domain (Doc2) family of presynaptic calcium-binding proteins as potential regulators of spontaneous (Groffen et al., 2010; Pang et al., 2011) and asynchronous (Yao et al., 2011) release, although they may or may not function as calcium sensors in this process. We obtained shRNA knockdown constructs directed against all four isoforms of the Doc2 family of proteins (Doc2A, Doc2B, Doc2G, and rabphilin) and proceeded to evaluate whether this alternative means to selectively reduce spontaneous release leads to synaptic scaling. With this method, we observed significant knockdown of Doc2A (47.9 $\pm 11.1 \%$ reduction; Bonferroni corrected, $p<0.05)$, Doc2B (63.8 $\pm 9.5 \%$ reduction; Bonferroni corrected, $p<0.05)$, and rabphilin $(62.5 \pm 9.7 \%$ reduction; Bonferroni corrected, $p<0.05$ ) compared with control-infected neurons using $\mathrm{qRT}$-PCR and a nonsignificant knockdown of Doc2G $(24.0 \pm 20.0 \%$ reduction; uncorrected, $p=0.16$; Student's paired $t$ test; $n=4$ independent cultures). This Doc2 and rabphilin knockdown (Doc/Rph KD) strategy has been previously validated (Pang et al., 2011) and reduces the likelihood of potential functional compensation among the closely related Doc2 proteins.

To test the effects of Doc2 4KD on synaptic strength, we measured AMPA-mEPSCs in cultured rat hippocampal neurons in

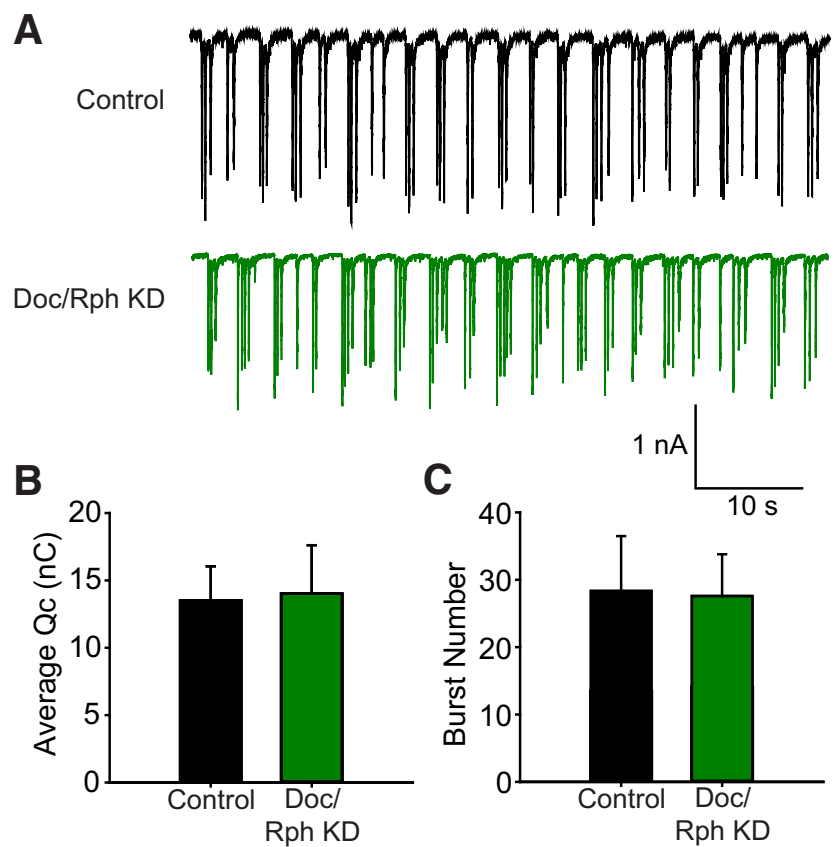

Figure 4. Network-driven excitatory activity is not altered by loss of Doc2-like proteins $\boldsymbol{A}$, Representative traces of network-driven EPSCs from Control and Doc/Rph KD neurons in rat hippocampal cultures. $\boldsymbol{B}$, No significant difference was observed in average total charge transfer (Qc) between Control and Doc/Rph KD neurons from 3 min of recording ( $p=0.91$; Student's $t$ test; $n=12$ neurons from five independent cultures). $\boldsymbol{C}$, No significant difference was observed in average EPSC burst number between Control and Doc/Rph KD neurons analyzed in $\boldsymbol{B}$ ( $p=$ 0.94; Student's $t$ test).

the presence of TTX to block action potentials. Representative traces of AMPA-mEPSC recordings from control and Doc2/rabphilin knockdown (Doc/Rph KD) neurons are shown in Figure $1 A$, and cumulative probability analysis of AMPA-mEPSC interevent intervals revealed a significant decrease in AMPA-mEPSC event frequency concentrated in the low range of interevent intervals (Fig. 1B). Furthermore, Doc/Rph KD elicited an increase in AMPA-mEPSC amplitude compared with control neurons (Fig. 1C), and a rank-order plot of these events indicated a multiplicative increase of $289 \%$ in Doc/Rph KD neurons (Fig. 1D), consistent with a robust homeostatic synaptic scaling response. When shRNA-resistant Doc2B was coexpressed with Doc/Rph $\mathrm{KD}$, both the decreased frequency and the increased amplitude of AMPA-mEPSCs were rescued to control levels (Fig. 2). Together, these data support prior work showing that Doc2 protein knockdown reduces the frequency of spontaneous neurotransmission at excitatory synapses (Groffen et al., 2010; Pang et al., 2011) and additionally suggests that this reduction in frequency coincides with an increase in synaptic strength.

\section{Knockdown of Doc2 isoforms does not scale inhibitory synapses}

In a previous study, reduced spontaneous release via SNARE protein loss induced strong scaling at excitatory synapses but no significant scaling at inhibitory synapses (Crawford et al., 2017). To determine whether the changes in synaptic strength discovered in excitatory synapses after Doc/Rph KD were also exhibited in inhibitory synapses, we recorded GABA-mIPSCs in control and Doc2/rabphilin KD neurons (Fig. 3A). Similar to AMPAmEPSCs, a loss of Doc2-like proteins significantly decreased GABA-mIPSC frequency (Fig. 3B). No significant differences, however, were found between GABA-mIPSC amplitudes in Doc/ 
Rph KD neurons and control neurons (Fig. 3C). These data indicate that, although one or more of the Doc2-like proteins controls a portion of spontaneous vesicle release at inhibitory synapses, this alteration in event frequency does not coincide with a change in inhibitory event amplitude. This suggests that excitatory and inhibitory synapses exhibit segregated mechanisms for synaptic scaling.

\section{Knockdown of Doc2 isoforms does not alter action potential-driven excitatory activity}

The altered excitatory synaptic strength produced by Doc/Rph KD that we observed could potentially translate into altered network activity due to increased sensitivity at individual synapses. To test whether inherent action potential-driven activity is altered after Doc/Rph KD, we measured EPSCs from cultured neurons in the absence of TTX (Fig. 4A). We observed no discernible differences between control and Doc/Rph KD cultures in total charge transfer over $3 \mathrm{~min}$ of recording or in the number of EPSC bursts (Fig. $4 B, C$ ).

To better define the specificity of the effect of Doc/Rph KD on release mode, we next measured evoked EPSC responses from control and Doc/Rph KD neurons using 50 and $100 \mathrm{~ms}$ interstimulation intervals. There were no differences between control and Doc/Rph KD neurons in paired pulse ratio at either interval ( $50 \mathrm{~ms}$ : control $=1.19 \pm 0.26, \mathrm{Doc} / \mathrm{Rph} \mathrm{KD}=0.88 \pm 0.10$; $n=9$ control and 10 Doc/Rph KD neurons; $p=0.25$, Student's unpaired $t$ test; $100 \mathrm{~ms}$ : control $=0.96 \pm 0.15$, Doc/Rph KD $=$ $0.94 \pm 0.03 ; n=12$ control and 13 Doc/Rph KD neurons; $p=$ 0.91 , Student's unpaired $t$ test). Additionally, eEPSC amplitudes were measured from the same recordings using the first pulse in the trains, and no significant difference was observed between control and Doc/Rph KD neurons (control $=1.71 \pm 0.32 \mathrm{nA}$, Doc/Rph KD $=2.05 \pm 0.32 \mathrm{nA} . p=0.46$, Student's unpaired $t$ test). These results suggest that the effects on quantal amplitude due to loss of the Doc2 family of proteins are largely specific to spontaneous events and support the notion that spontaneous and evoked postsynaptic responses rely on distinct downstream signaling pathways. We conclude that action potential-driven neurotransmitter release is not substantially altered at excitatory synapses after Doc/Rph KD.

\section{eEF2 signaling is important for scaling excitatory} synaptic strength

Presynaptic loss of the spontaneous neurotransmission-associated v-SNAREs vtila and VAMP7 leads to altered postsynaptic signaling through the eEF2 pathway, which is vital for the subsequent synaptic scaling effect in that system (Crawford et al., 2017). We next tested whether the spontaneous glutamate release regulated by Doc2-like proteins signals to postsynaptic eEF2 kinase in a way similar to vtila and VAMP7. We first performed luminal synaptotagmin antibody uptake experiments in cultured neurons in the presence of TTX to gauge the amount of spontaneous neurotransmission occurring over $15 \mathrm{~min}$. After fixation, we then immunostained for phosphorylated eEF2. Representative images from control and Doc/Rph KD neurons are shown in Figure $5 A$, and white arrows in each panel indicate typical punctate regions of interest that we examined. We measured the antibody intensity levels for both proteins in these regions and found that phospho-eEF2 staining and luminal synaptotagmin antibody uptake were both decreased in Doc/Rph KD synapses (Fig. 5B,C). Additionally, neuronal homogenates from control and Doc/Rph KD cultures were subjected to immunoblotting with antibodies against total eEF2, phospho-eEF2, and the loading control protein GDI (Fig. 5D). A significant reduction in the phospho/total eEF2 ratio was seen in Doc/Rph KD neurons compared with control neurons (43\% reduction; $p=0.0001$; Student's unpaired $t$ test; $n=13-17$ independent cultures). Together, these data confirm the decrease in spontaneous neurotransmission previously observed at synapses after Doc/Rph KD and further suggest that levels of eEF2 phosphorylation are also decreased.

To determine whether the decrease in phosphorylated eEF2 after Doc/Rph KD indicates a requirement for eEF2K signaling in the synaptic scaling we observe, we measured AMPA-mEPSCs in neurons cultured from $e E F 2 K$ knock-out $(\mathrm{KO})$ mice or their littermate controls (Fig. 6A). Doc/Rph KD produced a significant increase in interevent interval in both $e E F 2 \mathrm{~K} \mathrm{KO}$ and $e E F 2 \mathrm{~K}$ wildtype (WT) neurons compared with control neurons (Fig. 6B), indicating a decrease in AMPA-mEPSC event frequency. Loss of eEF2K alone, however, did not significantly alter the interevent interval from control neurons, which is similar to results from prior experiments (Crawford et al., 2017). Similarly, loss of 
A

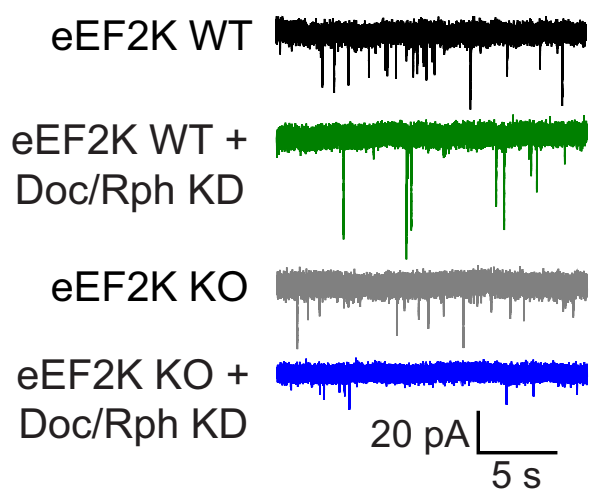

B AMPA-mEPSC Frequency

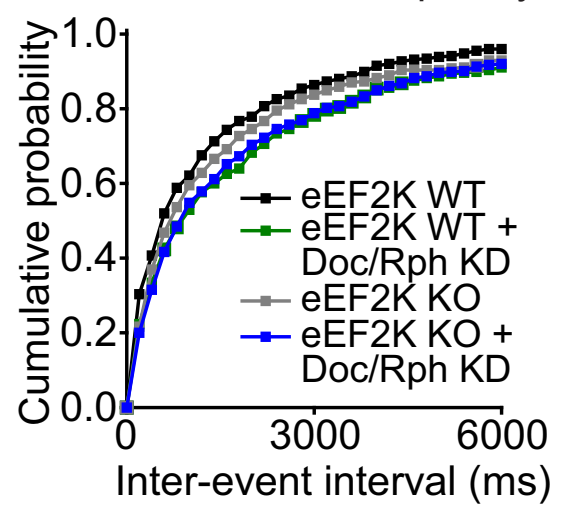

C AMPA-mEPSC Amplitude

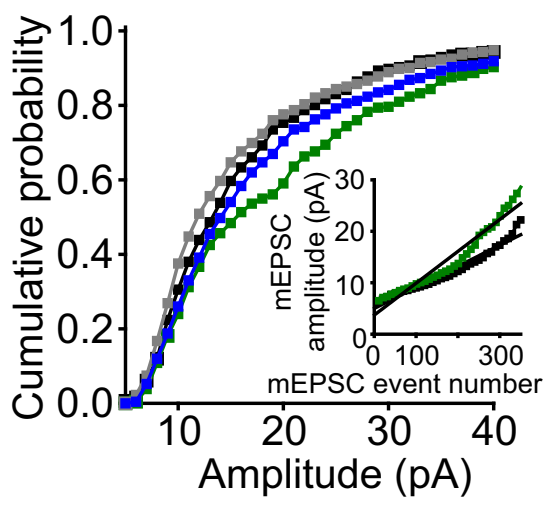

Figure 6. Synaptic scaling of AMPA-mEPSC amplitudes after loss of Doc2-like proteins requires eEF2 kinase. A, Representative traces of AMPA-mEPSC recordings in control-treated or Doc/Rph KD neurons in hippocampal cultures from eEF2K KO mice or their littermate controls (eEF2K WT). B, Cumulative probability histograms of AMPA-mEPSC interevent intervals from control-treated or Doc/Rph KD neurons from eEF2K KO mice or their littermate controls (eEF2K WT). Doc/Rph KD neurons from both eEF2K KO and eEF2K WT exhibited significantly increased interevent intervals compared with control-treated neurons, but control-treated eEF2K KO neurons did not differ significantly from eEF2KWT (eEF2KWT vs eEF2KWT + Doc/Rph KD: Bonferroni-corrected, $p<0.005$; eEF2KWT vs eEF2KKO + Doc/Rph KD: Bonferroni-corrected, $p<0.05$; eEF2KWT vs eEF2K KO: uncorrected, $p>0.05$; Kolmogorov-Smirnov test; $n=17$ neurons from five independent cultures). $\boldsymbol{B}$, Symbol legend refers to both $\boldsymbol{B}$ and $\boldsymbol{C}$. C, Cumulative probability histograms of AMPA-mEPSC amplitudes from data analyzed in $\boldsymbol{B}$. Doc/Rph KD neurons from eEF2KWT mice exhibited significantly increased AMPA-mEPSC amplitudes compared with control-treated neurons, but control-treated and Doc/Rph KD neurons from eEF2K KO mice did not differ significantly from eEF2KWT (eEF2KWT vs eEF2KWT + Doc/Rph KD: Bonferroni-corrected, $p<0.001$; eEF2KWT vs eEF2KKO + Doc/Rph KD: uncorrected, $p>0.05$; eEF2KWT vs eEF2K KO: uncorrected, $p>0.05$; Kolmogorov-Smirnov test). Inset, Rank-order plot of mEPSC amplitudes from control treated (black squares) and Doc/Rph KD (green squares) eEF2KWT neurons. The slopes of the linear fits of the two curves indicate a multiplicative increase (51\% scaling) of AMPA-mEPSC amplitudes in Doc/Rph KD neurons compared with control. To improve the linear fits of the slope, additional analyses of the initial portion (mEPSCs $0-200$ ) and later portion (mEPSCs 200 -350) of the plot were conducted and showed a 44\% and 53\% increase in mEPSC amplitudes, respectively, following Doc/Rph KD.

eEF2K alone did not significantly alter the amplitude of AMPAmEPSC events compared with control neurons, but it did prevent the increase in AMPA-mEPSC amplitude observed after Doc/ Rph KD (Fig. 6C). Finally, a rank-order plot of mEPSC amplitudes recorded from $e E F 2 K$ WT control or Doc/Rph neurons indicates a multiplicative increase in amplitude of $51 \%$ after Doc/ Rph KD (Fig. 6C, inset). These data suggest that eEF2K signaling does not interfere with basal parameters of AMPA-mEPSC events in our model, but eEF2K is contributing to the increased efficacy of excitatory synapses after loss of Doc2-like proteins.

\section{Discussion}

Doc2-like proteins are known to modulate spontaneous and asynchronously evoked neurotransmission, but it was previously unclear whether the neurotransmission controlled by these proteins also influences synaptic strength. We found that knockdown of multiple Doc2-like proteins reduces the frequency of excitatory spontaneous events and increases the efficacy of individual synapses. Although the frequency of inhibitory spontaneous neurotransmission was also reduced, inhibitory synapses did not exhibit increased synaptic efficacy, suggesting that excitatory and inhibitory synapses are regulated separately. Rescue of excitatory events was successful with Doc2B overexpression, implicating this Doc2-like protein as a major driver of this process. Despite the effects of quadruple knockdown on spontaneous neurotransmission, action potential-driven excitatory activity appears normal, suggesting that this molecular manipulation selectively impairs subsets of neurotransmission modes. Additionally, loss of Doc2-like proteins reduced eEF2 phosphorylation, and the resulting increase in excitatory synaptic strength depends on eEF2 kinase activity.

Previous reports indicate that Doc2A and Doc2B are involved in spontaneous (Groffen et al., 2010; Pang et al., 2011) and asynchronous (Sakaguchi et al., 1999; Yao et al., 2011) neurotransmitter release. Like previous studies (Groffen et al., 2010; Pang et al., 2011), we found that loss of Doc2-like proteins reduced the fre- quency of excitatory spontaneous events in a manner that could be rescued by Doc2B overexpression. While our data are largely consistent with previous reports, we found a larger effect of quadruple knockdown on AMPA-mEPSC amplitudes than prior studies (Pang et al., 2011), but the direction of the change agrees despite the difference in magnitude. Interestingly, both Doc2A (Yao et al., 2011) and Doc2B (Groffen et al., 2010) have been shown to affect neural network activity, whereas we found no effect of quadruple knockdown on endogenous network activity in cultured hippocampal neurons. This discrepancy may be due to the different cell types tested or the potential compensatory effects of other Doc2-like proteins or even synaptotagmin1, a calcium sensor that may compensate for Doc2-like proteins in synchronous release (Yao et al., 2011), in each study. Further work is needed to clarify how these presynaptic proteins cooperate in regulating neurotransmission.

Loss of the Doc2 family of proteins elicited similar synaptic regulation as observed after loss of vtila and VAMP7, two $\mathrm{v}$-SNARE proteins that selectively drive spontaneous neurotransmission (Hua et al., 2011; Ramirez et al., 2012; Bal et al., 2013). Although it is unclear whether Doc2-like proteins act in conjunction with vtila or VAMP7 or whether these proteins regulate the same subset of synaptic vesicles, loss of Doc2-like proteins and loss of vtila and VAMP7 both reduce the number of spontaneous release events while increasing their efficacy in an eEF2dependent manner (Crawford et al., 2017). This is somewhat surprising as loss of canonical release machinery, like synaptobrevin2 (Schoch et al., 2001) and synaptotagmin1 (Geppert et al., 1994; Xu et al., 2009), decreases or increases the frequency of spontaneous events, respectively. Yet these manipulations do not alter postsynaptic efficacy, indicating some divergence in signaling downstream of the canonical machinery mediated release versus release mediated by Doc2 or vtila and VAMP7. Both Doc2A (Yao et al., 2011) and VAMP7 (Scheuber et al., 2006) also modulate asynchronous evoked release in addition to spontane- 
ous neurotransmission, which may suggest that there is some overlap between these two forms of release. At least one study argues that these proteins regulate spontaneous neurotransmission in a differential manner. In that study, Reelin-induced increases in spontaneous neurotransmission were dependent on VAMP7 but not the Doc2 protein family (Bal et al., 2013). Thus, there appears to be some divergence in the release-mode specific functions of these proteins.

Together, these results suggest that spontaneous neurotransmission modulated by Doc2-like proteins regulates synaptic efficacy. This regulation relies on eEF2 signaling, which may have important implications due to eEF2's role in the fast-acting antidepressant actions of ketamine treatment (Autry et al., 2011; Nosyreva et al., 2013). Additionally, these data reinforce previous work that suggests spontaneous and synchronously evoked neurotransmission communicate independently to the postsynaptic cell and induce separate signaling cascades (Reese and Kavalali, 2015; Reese and Kavalali, 2016). Although under physiological conditions these forms of neurotransmission likely cooperate to transfer reliable information throughout the nervous system, it is becoming increasingly clear that they maintain a significant degree of functional segregation.

\section{References}

Adachi M, Monteggia LM (2014) Decoding transcriptional repressor complexes in the adult central nervous system. Neuropharmacology 80:45-52. CrossRef Medline

Andreae LC, Fredj NB, Burrone J (2012) Independent vesicle pools underlie different modes of release during neuronal development. J Neurosci 32: 1867-1874. CrossRef Medline

Antonin W, Riedel D, von Mollard GF (2000) The SNARE vtila-beta is localized to small synaptic vesicles and participates in a novel SNARE complex. J Neurosci 20:5724-5732. Medline

Aoto J, Nam CI, Poon MM, Ting P, Chen L (2008) Synaptic signaling by all-trans retinoic acid in homeostatic synaptic plasticity. Neuron 60:308320. CrossRef Medline

Autry AE, Adachi M, Nosyreva E, Na ES, Los MF, Cheng PF, Kavalali ET, Monteggia LM (2011) NMDA receptor blockade at rest triggers rapid behavioural antidepressant responses. Nature 475:91-95. CrossRef Medline

Bal M, Leitz J, Reese AL, Ramirez DM, Durakoglugil M, Herz J, Monteggia LM, Kavalali ET (2013) Reelin mobilizes a VAMP7-dependent synaptic vesicle pool and selectively augments spontaneous neurotransmission. Neuron 80:934-946. CrossRef Medline

Crawford DC, Kavalali ET (2015) Molecular underpinnings of synaptic vesicle pool heterogeneity. Traffic 16:338-364. CrossRef Medline

Crawford DC, Ramirez DM, Trauterman B, Monteggia LM, Kavalali ET (2017) Selective molecular impairment of spontaneous neurotransmission modulates synaptic efficacy. Nat Commun 8:14436. CrossRef Medline

Fong MF, Newman JP, Potter SM, Wenner P (2015) Upward synaptic scaling is dependent on neurotransmission rather than spiking. Nat Commun 6:6339. CrossRef Medline

Gaffaney JD, Xue R, Chapman ER (2014) Mutations that disrupt Ca(2)(+)binding activity endow Doc2beta with novel functional properties during synaptic transmission. Mol Biol Cell 25:481-494. CrossRef Medline

Geppert M, Goda Y, Hammer RE, Li C, Rosahl TW, Stevens CF, Südhof TC (1994) Synaptotagmin I: a major $\mathrm{Ca}^{2+}$ sensor for transmitter release at a central synapse. Cell 79:717-727. CrossRef Medline

Groffen AJ, Martens S, Díez Arazola R, Cornelisse LN, Lozovaya N, de Jong AP, Goriounova NA, Habets RL, Takai Y, Borst JG, Brose N, McMahon HT, Verhage M (2010) Doc2b is a high-affinity $\mathrm{Ca}^{2+}$ sensor for spon- taneous neurotransmitter release. Science 327:1614-1618. CrossRef Medline

Hogins J, Crawford DC, Jiang X, Mennerick S (2011) Presynaptic silencing is an endogenous neuroprotectant during excitotoxic insults. Neurobiol Dis 43:516-525. CrossRef Medline

Hua Z, Leal-Ortiz S, Foss SM, Waites CL, Garner CC, Voglmaier SM, Edwards RH (2011) v-SNARE composition distinguishes synaptic vesicle pools. Neuron 71:474-487. CrossRef Medline

Kavalali ET (2015) The mechanisms and functions of spontaneous neurotransmitter release. Nat Rev Neurosci 16:5-16. CrossRef Medline

Kavalali ET, Klingauf J, Tsien RW (1999) Activity-dependent regulation of synaptic clustering in a hippocampal culture system. Proc Natl Acad Sci U S A 96:12893-12900. CrossRef Medline

McKinney RA, Capogna M, Dürr R, Gähwiler BH, Thompson SM (1999) Miniature synaptic events maintain dendritic spines via AMPA receptor activation. Nat Neurosci 2:44-49. CrossRef Medline

Muzerelle A, Alberts P, Martinez-Arca S, Jeannequin O, Lafaye P, Mazié JC, Galli T, Gaspar P (2003) Tetanus neurotoxin-insensitive vesicle-associated membrane protein localizes to a presynaptic membrane compartment in selected terminal subsets of the rat brain. Neuroscience 122:59-75. CrossRef Medline

Nosyreva E, Szabla K, Autry AE, Ryazanov AG, Monteggia LM, Kavalali ET (2013) Acute suppression of spontaneous neurotransmission drives synaptic potentiation. J Neurosci 33:6990-7002. CrossRef Medline

Pang ZP, Bacaj T, Yang X, Zhou P, Xu W, Südhof TC (2011) Doc2 supports spontaneous synaptic transmission by a $\mathrm{Ca}(2+)$-independent mechanism. Neuron 70:244-251. CrossRef Medline

Raingo J, Khvotchev M, Liu P, Darios F, Li YC, Ramirez DM, Adachi M, Lemieux P, Toth K, Davletov B, Kavalali ET (2012) VAMP4 directs synaptic vesicles to a pool that selectively maintains asynchronous neurotransmission. Nat Neurosci 15:738-745. CrossRef Medline

Ramirez DM, Andersson S, Russell DW (2008) Neuronal expression and subcellular localization of cholesterol 24-hydroxylase in the mouse brain. J Comp Neurol 507:1676-1693. CrossRef Medline

Ramirez DM, Khvotchev M, Trauterman B, Kavalali ET (2012) vtila identifies a vesicle pool that preferentially recycles at rest and maintains spontaneous neurotransmission. Neuron 73:121-134. CrossRef Medline

Reese AL, Kavalali ET (2015) Spontaneous neurotransmission signals through store-driven $\mathrm{Ca}^{2+}$ transients to maintain synaptic homeostasis. eLife 4.

Reese AL, Kavalali ET (2016) Single synapse evaluation of the postsynaptic NMDA receptors targeted by evoked and spontaneous neurotransmission. eLife 5.

Sakaguchi G, Manabe T, Kobayashi K, Orita S, Sasaki T, Naito A, Maeda M, Igarashi $\mathrm{H}$, Katsuura G, Nishioka H, Mizoguchi A, Itohara S, Takahashi T, Takai Y (1999) Doc2alpha is an activity-dependent modulator of excitatory synaptic transmission. Eur J Neurosci 11:4262-4268. CrossRef Medline

Scheuber A, Rudge R, Danglot L, Raposo G, Binz T, Poncer JC, Galli T (2006) Loss of AP-3 function affects spontaneous and evoked release at hippocampal mossy fiber synapses. Proc Natl Acad Sci U S A 103:1656216567. CrossRef Medline

Schoch S, Deák F, Königstorfer A, Mozhayeva M, Sara Y, Südhof TC, Kavalali ET (2001) SNARE function analyzed in synaptobrevin/VAMP knockout mice. Science 294:1117-1122. CrossRef Medline

Sutton MA, Ito HT, Cressy P, Kempf C, Woo JC, Schuman EM (2006) Miniature neurotransmission stabilizes synaptic function via tonic suppression of local dendritic protein synthesis. Cell 125:785-799. CrossRef Medline

Xu J, Pang ZP, Shin OH, Südhof TC (2009) Synaptotagmin-1 functions as a $\mathrm{Ca}^{2+}$ sensor for spontaneous release. Nat Neurosci 12:759-766. CrossRef Medline

Yao J, Gaffaney JD, Kwon SE, Chapman ER (2011) Doc2 is a $\mathrm{Ca}^{2+}$ sensor required for asynchronous neurotransmitter release. Cell 147:666-677. CrossRef Medline 